\title{
REVIEW ARTICLE POTENTIAL ROLE OF GUT MICROBIOTA IN OBESITY AND CARDIOVASCULAR DISEASES
}

\author{
Sana Ashiq ${ }^{1}$, Kanwal Ashiq ${ }^{2}$ \\ ${ }^{1}$ Centre for Applied Molecular Biology (CAMB), University of the Punjab, Lahore-Pakistan, ${ }^{2}$ Faculty of Pharmaceutical Sciences, \\ Superior College Lahore, Superior University 17-km Lahore, Pakistan
}

From the past several decades to the present, obesity and cardiovascular diseases emerge as a major cause of mortality and morbidity, ultimately imposing a huge economic burden globally. In humans, a healthy gut microbiota metabolizes several indigestible dietary components which maintain host immune homeostasis, but an imbalanced host-microbiota is associated with various metabolic disorders including cardiovascular diseases and obesity. In this present review, we critically analyze the literature which provides better insight into the mechanistic link of gut microbiota with obesity and cardiovascular diseases. There is strong e vidence that suggests the potential role of the gut microbiota in metabolic disorders including cardiovascular diseases and obesity. In addition, the different studies report that gut microbes can play a promising role in the management of obesity and the treatment of cardiovascular diseases. In the future, multidisciplinary approaches and advancements in culturing and molecular biology techniques can provide us a better understanding.

Keywords: Microbiota, Obesity, Cardiovascular diseases, Gut, trimethylamine N-oxide

Citation: Ashiq S, Ashiq K. Potential Role of Gut Microbiota in Obesity and Cardiovascular Diseases. Pak Heart J. 2021;54(03):207-213. DOI: https://doi.org/10.47144/phj.v54i3.2129

\section{INTRODUCTION}

The term intestinal microbiota refers to the pool of various microorganisms present in the gastrointestinal tract (GIT) of the humans. ${ }^{1}$ This gut microbiota shapes the physiology of the host immune system through assimilation and digestion of nutrients, host tissue maturation, and inhibit a deleterious appearance of opportunistic pathogens. ${ }^{2}$ The human metabolism is influenced by the intestinal microbiome. This review critically discusses the various aspects of gut microbiota with human pathophysiology pathways and their role in the pathogenesis of cardiovascular diseases (CVDs) and obesity.

Globally, a high number of pregnant women are obese or overweight, which increases two-to-five-fold greater risk of intergenerational obesity. A novel hypothesis suggest that the obesogenic microbiota is considered as one of the major causative agents in newborns during its transmission from mother to fetus. ${ }^{3}$ The low-level transmission of microorganism through the placenta from mother to fetus has been reported. The nonpathogenic bacteria from the Fusobacteria, Tenericutes, Bacteroidetes, Firmicutes and Proteobacteria have been detected in healthy newborn babies' meconium. ${ }^{4}$ In infants, the composition of gut bacteria is influenced by the several factors such as mode of delivery at birth [cesarean section [CS] vs. vaginal delivery], feeding and weaning. ${ }^{5}$
The impact of CS birth on microbiota has been associated with obesity or high infection rate due to adverse immune development as compared to the vaginal delivery ${ }^{6}$ intrapartum antibiotics may affect the fetus health status as it decreased the level of Bifidobacterium, Bacteroides and Blautia. ${ }^{7}$ In some countries the highest incidence reaching up to $40.5 \%$ is alarming. ${ }^{8}$ Azad et al further revealed that difference in cesarean delivered infants gut microbiota may be due to the [elective vs. emergent] suggestion that it is associated with underlying medical conditions rather than a surgical procedure. Till now the presence of such confounding factors remains unexplored and have not been established. ${ }^{9}$

The breast milk keeps the high number of Bifidobacterium in the gut and lowered the other bacteria Enterobacteriaceae, Streptococcaceae and Lactobacillaceae as compared to the mixed feeding which alters this percentage of microorganisms. ${ }^{10}$ Due to formula feeding, the increased number of Firmicutes and Bacteroidetes was reported. These alterations continued for the first three years of age until a well-balanced microbiota and host states called as Enterotypes is established which is the stable microbiota. ${ }^{11}$ In adult, human gut is composed of approximately hundred trillion bacteria and mainly consist of the three phyla: Actinobacteria, Firmicutes and Bacteroidetes. ${ }^{12}$ Several reports suggested that the gut microbiota is specific to every individual. Thus, difference in composition of gut microbiota and 
functional genes associated with different metabolic disorders. ${ }^{13}$

To date, various studies reported the potential role of gut microbiota in obesity and cardiovascular disorders but, the results remain inconsistent, hence, it is the need of the hour to analyze all the literature available that provide us the more definite results about the mechanistic link of gut microbiota with metabolic disorders and treatment strategies.

The purpose of the current review is to critically analyze the role of the gut microbiota in cardiovascular diseases and obesity. We also reviewed the advanced laboratory analysis techniques and therapeutic strategies

\section{METHODOLOGY}

The current literature was selected from the November 2019 to April 2020. A detailed search was done by using different databases including Google and Bing, Google Scholar and Medline/PubMed. Moreover, to ensure the credibility only indexed journal articles was included for study.

Impact of host genetics on gut microbiota: Host genetics have shown the role in affecting microbiome composition and individual bacterial taxa. ${ }^{14}$ The classic approach used to differentiate the effects of shared environment from those of shared genetics is comparing the dissimilarity between the groups of dizygotic twins and monozygotic twins. Each pair of dizygotic and monozygotic twins revealed the variation in genetic similarity $50 \%$ and $100 \%$ similarity while both types share the same environment. There are several reports which confirmed the significant heritability of individual bacterial taxa and overall microbiome composition. ${ }^{14}$

Microbiome genome wide association studies (mGWAS) are the cohort studies from the same individual to investigate how the composition of microbiome and host genetics affect health and disease which combine whole genome sequencing (WGS) or human genome typing with analysis of microbiome (metagenomic sequencing or 16 ribosomal RNA (rRNA). In 2017 mGWAS, confirmed the high number of associations between the human host genetic variants and gut microbiome. Moreover, recently the family Methanogens and christensenellaceae also identified as the heritable bacterial taxa by using mGWAS. ${ }^{15}$ Awany et al. in 2019 report the association between the gut microbiome to the wide range of complex diseases including cardiovascular diseases, diabetes, obesity, inflammatory bowel diseases, weight gain and asthma. ${ }^{15}$

Impact of environmental factors on gut microbiota: The maternal environment is considered as one of the earliest factors which affects the gut microbiota composition. The health status of the offspring is affected by the maternal metabolic, nutritional, and physiological states that ultimately leads to abnormalities during developmental stages of the fetus as well as after birth increased the risk of noncommunicable diseases among the newborns. ${ }^{16}$ There are different experiments performed on the animals which proved that the presence of the microbiota in the fetus is from the pregnant woman. Moreover, mother weight gain during pregnancy can also affect the microbiota composition as compared to the recommended weight gain mothers' infants. The current evidence suggests that the at six-month high abundance of Clostridium histolyticum and at onemonth lower number of the bacteriodes-prevotella group due to obesity in mothers. ${ }^{17}$ The gestational age at birth also results in various alteration in gut microorganisms as Fouhy et al. in 2019 reports that the preterm infants have decreased the number of the Bifidobacterium as compared to the full-term infants. Profiles of microbiota are also characteristic in children depends on gestational maturity and age as the preterm and full-term delivery is characterized by at year one Lactobacillus and Bacteroides at year two Streptococcus and Parabacteroides while at year three Carnobacterium and Christensenellaceae respectively. ${ }^{18}$

Gut microbiota composition: Due to the diversity, complexity, and variation among individual results in different microbial taxa which thus precise and complete composition of gut microorganisms remains a challenge. The core microbiome concept by using the culture-based studies states that the Escherichia coli is a core of stable colonizer around which other microbes fluctuates slowly. While in comparison sequencing based studies report the microorganisms in the gut are hypervariable in population, which ultimately confirmed the high specificity of final composition and the concept of core microbiome is less accepted. ${ }^{4}$

The human gut microbiota is composed of transient or allochthonous microorganism and indigenous microorganisms also called as autochthonous. It is comprised on the Eubacteria, Archaea and Eukarya which shows the complex, trophic relationship with each other and host. ${ }^{19}$ Figure 1 explains the factors affecting the gut microbiota composition. ${ }^{19}$ 
In healthy adult, gut microbiota is dominated by the phyla Bacteroidetes and Firmicutes followed by the minor constituents of the Verrucomicrobia and Actinobacteria. The species occurring in the gut microbiota are described further in Figure $2 .{ }^{20}$ In archaeal population the dominant microorganism is M. smithii which is the hydrogen utilizing methanogen. ${ }^{4}$

Gut microbiota physiological role: In humans, the gut microbiota plays an essential role during the metabolic process, particularly to those which are linked to dietary metabolism. Over three million genes encodes by the microbiome which encodesa large number of metabolites which influences many functions of the host, including various aspects of host health such as metabolic, neurobehavioral traits and immune system. Other key roles are those associated with the short chain fatty acid metabolism, improved lipid metabolism, insulin signaling, decreases kidney toxin [indoxil sulphate], increase antioxidant production, conversion of bile acids to bile salts and vitamin synthesis. ${ }^{21}$ Dysbiosis or imbalance in the gut microbiota composition can cause obesity, cardiovascular diseases, diabetes, and metabolic syndrome or in other case it may cause increased susceptibility to infections in the intestines due to its protective role in GIT in which it inhibits the colonization of opportunistic or pathogenic bacteria in the gut. ${ }^{13}$ The gut normal flora may perturb due to overuse or misuse of broad spectrum antibiotics, thus causing the recurrent infections, obesity, CVDs or may lead to the emergence of multi-drug resistant [MDR] bacteria. Novel therapies are being developed to complement antibiotic therapies attempting either re-establish the beneficial effects of commensals communities to the host together with selectively target the target pathogens without disturbing the gut microbiota. ${ }^{22}$

Techniques/approaches used for the gut microbiota determination: There are several methods which are used for the gut microbiota composition from routine culture methods used in microbiology to the culture independent approaches. The details of each of these major approaches are discussed in detail, including every method use and limitations in the following section.

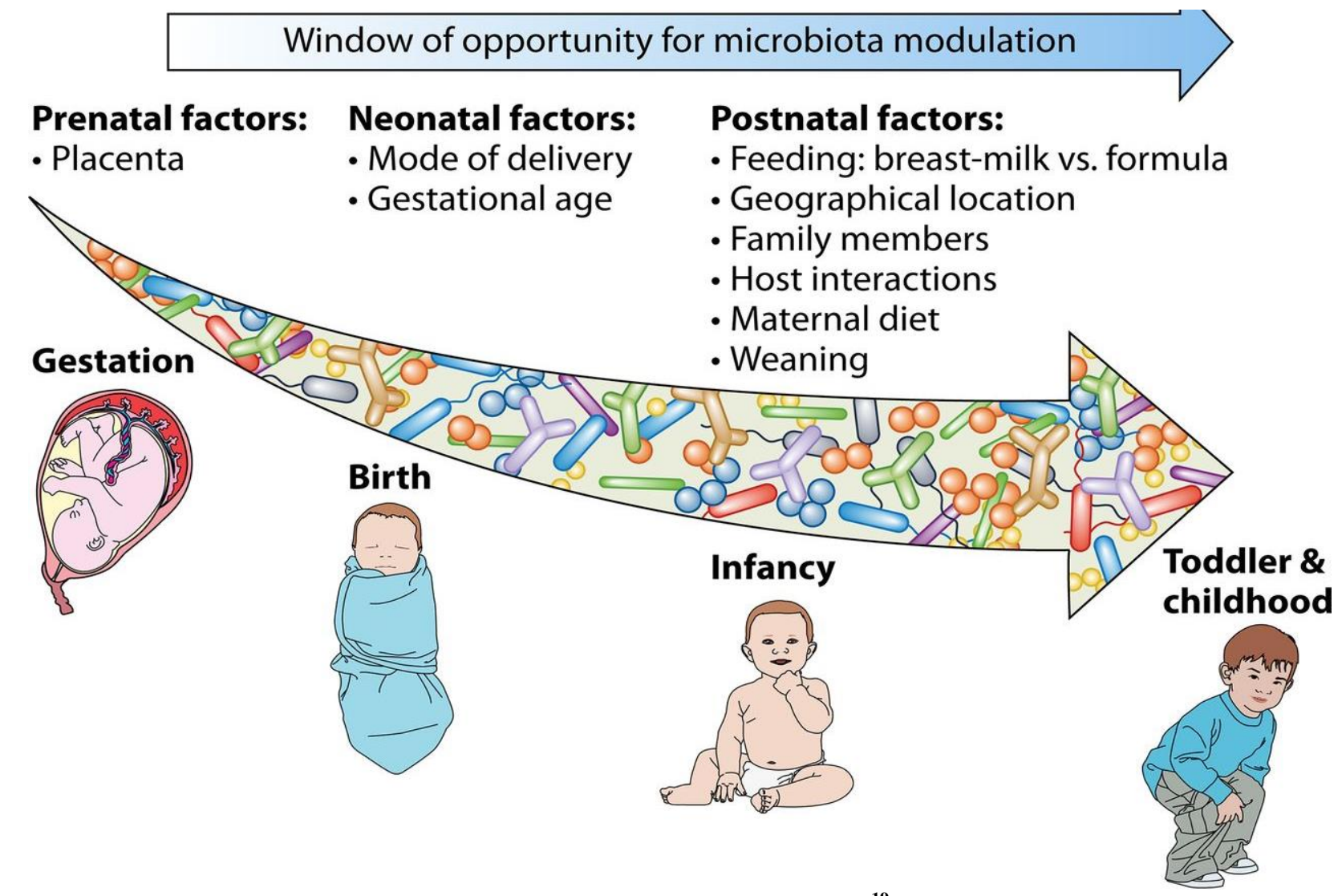

Figure 1: Major factors affecting the composition of the gut microbiota ${ }^{19}$ 


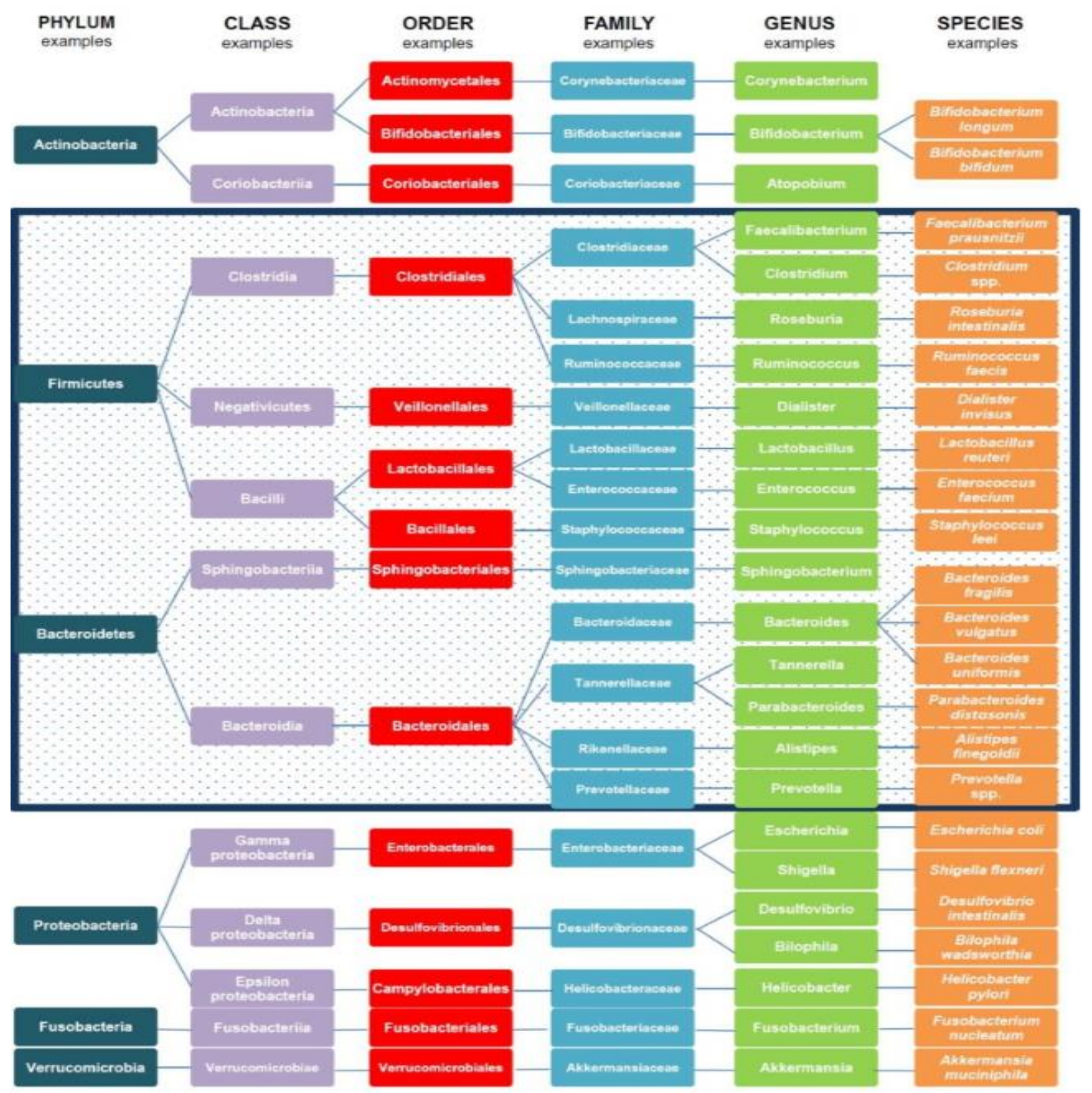

\section{Figure 2: Gut bacterial composition ${ }^{20}$}

Culture-based: The culture-based method uses the different culture media for isolation, identification, and characterization of the bacteria. But it provides limited insight due to the gap of knowledge regarding growth requirements $(\mathrm{pH}$, temperature, and selection of appropriate media) for gut microbiota thus only $10-20 \%$ of entire gut microbiota are easily cultured..$^{23}$

Metagenomics: The characterization of these microbiota appears a major challenge for the scientist around the globe due to the limitations of culturing techniques used in microbiology on the routine basis. The recent culture independent approaches or omics techniques has been revolutionizing the studies attempting to identify the composition of the gut microbiota.

Shotgun sequencing: Metagenome assembled genome is a method that enables the recovery of potential genomes by using the denovo assembly of shotgun metagenomic reads into counting sequences on the basis of tetraneucleotide frequency and sequence coverage and then place them into various bins. ${ }^{24}$ The shotgun metagenomics advantages include more specific 
functional and taxonomic classification of sequences, better resolution and discovery of novel bacterial genes and genomes but it is considered as expensive approach for GIT microbiota determination. ${ }^{25}$

16S RNA sequencing: Microbial profiling using $16 \mathrm{~S}$ rRNA gene is basically the method which involves the 9 different hypervariable region flanked by highly conserved deoxyribose nucleic acid sequence which are suitable site for primer binding. ${ }^{19}$ The targeted 16S RNA sequencing method provides more abundant data at the taxonomic level and phylogenetic relationship, but in comparison to the shotgun sequence it has low resolution during polymerase chain reaction (PCR) step. ${ }^{25}$

Metaproteomics: Metaproteomics identifies and detects the diverse proteins which further help to unravel the molecular mechanisms underlying health and disease pathogenesis, employed as biomarker during diagnosis which ultimately provides more strategies regarding disease prevention and management. But this approach includes major limitations regarding protein extraction and purification. ${ }^{26}$

Metatranscriptomics: The RNA sequencing of the gut microbiota provides insight into the biochemical activities of microbiota by quantifying the active gene microbial expression. And for all metabolically active organisms, it provides strain level taxonomic resolution. But it is more expensive and laborious due to the laboratory and depth in-silico analysis. ${ }^{27}$

Culturomics: The rapid identification of gut microbiota by using a high throughput culture approach is the application of culturomics. It consists of mass spectrometry or $16 \mathrm{~S}$ rRNA sequencing, which is used for the previous unculturable or unidentified bacterial species. $^{28}$

Role and composition of gut microbiota in obesity: The genetic spectrum of obesity is very complex and contributes a small proportion in obesity progression. A number of genetic factors implicated in the polygenic and monogenic forms of obesity. Genome wide association studies report significant role of single nucleotide polymorphisms in several genes (leptin, leptin receptors, fat mass and obesity associated gene (FTO)) in causing obesity. ${ }^{29}$ The gut microbiota composed of the five phyla which provides protection against a wide range of pathogenic or opportunistic bacteria. Thus, the disturbance in microbial equilibrium or dysbiosis is also associated with obesity, which is further confirmed by experimental studies in mice as results shows $50 \%$ less Bacteroidetes and high Firmicutes in ob/ob (genetically obese) mice as compared to lean or normal gut microbiota composition. ${ }^{30}$ The impact of gut microbiota on distant and local organs contribute to the pathogenesis of obesity. In local tissue, gut microbiota has a potential role in causing obesity as it increases in energy harvest from the diet, in colon stimulates gene reprogramming, and alters the bioactive molecules and polypeptide hormones released by enteroendocrine cells affecting the immune homeostasis and the intestinal barrier. These microorganisms can also communicate with host brain, liver, and adipose tissue. Gut microbiota through the fat signaling axis participate in the adipogenesis, and along with inflammation the lipopolysaccharide triggers an immune response. Through the activation of G-protein coupled receptor 41/43 (GRP41, and GRP43) short chain fatty acids (SCFAs) also participate in insulin mediated fat accumulation in adipocytes which further inhibits the lipolysis. Gut bioactive molecules and hormones can affect gut afferent neurons ultimately disturbing the gutbrain axis. The dysbiosis can also cause results disturbance in liver-gut axis, which include increased permeability of lipopolysaccharide, bile acids, and short chain fatty accumulation. ${ }^{31}$ In Pakistan, Ahmad et al. analyze the gut microbiota composition of obese individuals with type 2 diabetes mellitus by using the $16 \mathrm{~S}$ rRNA sequencing technique. The results of this study revealed the Firmicutes, Negativicutes, and Clostridia were predominant microorganisms in these patients. ${ }^{32}$

Composition and role of gut microbiota in cardiovascular diseases: Cardiovascular diseases are the group of disorders involving the heart and its blood circulation. In developing countries, its prevalence is the highest which poses a threat and huge burden to health care system. It is a multifactorial disorder involving both the genetic and environmental factor. Conventional risk factors are further divided into two types; modifiable and non-modifiable risk factors. Modifiable risk factors involve the smoking, sedentary lifestyle, obesity, stress, hypertension, and diabetes. Whereas the non-modifiable risk factors include gender, age, and family history. ${ }^{33}$

The genetic association involves the genes which regulate the pathways involve in pathophysiology such as lipid metabolism (LPL, LPA, LDR, APOs, CETP and ABCA1), inflammatory (interleukins), renin-angiotensin pathway (ACE and AGT), oxidative pathways (PONs), hormonal (ESR1) and endothelial integrity (NOS3). ${ }^{33}$ The evidence reported from different studies implicated the role of the intestinal microbiota in the pathogenesis of CVDs. Jin et al. elucidate that the alterations in the GIT microorganisms is strongly associated with the pathophysiology of CVDs as it is further confirmed by the DNA from different species of bacteria which is found different in the gut and atherosclerotic plaque of the same individual which further suggest gut microbiome potential role in CVDs. The high ratio of the Firmicutes/ Bacteroides is the major indicator marker for 
gut microbiota role in CVDs. The gut microbiota profile in CVDs includes a high number of Lactobacillus, Enterobacteriaceae, Streptococcus spp and decreased number of Roseburia. ${ }^{34}$ Miele and Co compared the microbiological profile of control (healthy) and cases (patients), the patients of CVDs shows the highest number of Collinsella and Proteobacteria as compared to normal healthy subjects. ${ }^{35}$

The novel analytical techniques such as metagenomic sequencing provides insight into the role of the gut microbiota in the pathophysiology of CVDs. The animal and human model further explains that the trimethylamine $\mathrm{N}$-oxide (TMAO) production from the liver from gut microbiota derived trimethylamine shows a potential role in the atherosclerosis. Moreover, the TMAO can enhance platelet activation and thrombosis. ${ }^{36}$ Recent evidence from the various research reports suggest that the leaky blood vessels are the primary source of the LPS which enters the blood circulation, which can further activate the cytokines and inflammation ultimately contributing a fundamental cause of the heart failure. Moreover, comparative case control studies further suggest that the patients who have the bacterial DNA in peripheral blood vessels show high levels of inflammatory markers. ${ }^{37}$ Indirect associations of gut microbiota with CVDs is by its modulation effects on other three modifiable risk factors, including diabetes, obesity and hyperlipidemia as there is a high number of scientific evidence suggesting that microbiota role in short chain fatty acid fermentation and due to endotoxins low grade inflammation. ${ }^{38}$

Therapeutic relevance of the gut microbiota: So far, we have discussed in detail about the intestinal microbiota and various factors that can influence its composition. There are several medical and surgical treatment that can also affect the function and relative composition of the gut microbes such as probiotics, fecal microbiota transplant (FMT), antibiotics, gastric bypass, prebiotics, and postbiotics.

The bacteriotherapy or FMT concept was first introduced in China to treat food poisoning or diarrhea. It refers to the healthy person fecal suspension in another person for the treatment of a particular disease. The major hypothesis for this FMT based cure is the fact that few metabolic phenotypes is transferred which is further confirmed by several experiments in animal models. In the recent studies, it is proven effective as it cures more than $80 \%$ cases of severe infection due to Clostridium difficile. ${ }^{39}$ Thus, given its potential, if some standard and legal protocols are optimized FMT could be an effective option as personalized medicine to treat CVDs, obesity, and other metabolic disorders.
Probiotics are living microbes which could be beneficial in subjects with the loss of these microbial groups as the gut microbiota play a key role in energy harvest and metabolism so its administration in another person can cure the disorder. In infants conventional use of probiotic beverages has been shown to improve its metabolism in humans as well as rodent models. For example, Bifidobacterium containing probiotics increase metabolism, gut barrier function, and reduce endotoxemia in animal models. ${ }^{40}$

Conclusions and future perspective: Taken together, the scientific studies so far find the gut microbiota to be a novel way of weight management and also appears to be promising in the treatment of cardiovascular diseases through its key role in energy harvest and metabolism, fatty acid oxidation, fat storage, modulating the levels of trimethylamine $\mathrm{N}$-oxide and inflammatory mechanisms. The strain dependent effects of microbial members in the intestinal microbiota revealed a very complex interactions within the members of microbiota as well as with human physiology and genotype. Although exact cause-effect relationship between gut microbiota with CVDs and obesity is still unclear, but in future due to rapid developments in the field of culturing (unculturable bacterial members) and genotyping may give us a clear picture of its real impact on host metabolism. Moreover, there is a need of multidisciplinary research to explicate the role of individual microorganisms, in specific circumstances and to devise strategies for fecal microbiota transplant, pharmacological or dietary interventions to modulate the gut microbes to treat metabolic disorders including cardiovascular diseases and obesity. In future, more studies regarding systematic reviews on gut microbiota can provide better insights about its role in metabolic disorders.

\section{AUTHORS' CONTRIBUTION}

SA and SK: Concept and design, data acquisition, interpretation, drafting, final approval, and agree to be accountable for all aspects of the work.

Conflict of interest: Authors declared no conflict of interest.

\section{REFERENCES}

1. Sánchez B, Delgado $\mathrm{S}$, Blanco-Míguez $\mathrm{A}$, Lourenço $\mathrm{A}$, Gueimonde M, Margolles A. Probiotics, gut microbiota, and their influence on host health and disease. Mol Nutr Food Res. 2017;61(1):1600240.

2. Splichal I, Donovan SM, Splichalova Z, Neuzil Bunesova V, Vlkova E, Jenistova V, et al. Colonization of germ-free piglets with commensal Lactobacillus amylovorus, Lactobacillus mucosae, and probiotic E. coli Nissle 1917 and their interference with Salmonella Typhimurium. Microorganisms. 2019;7(8):273.

3. Mueller NT, Shin H, Pizoni A, Werlang IC, Matte U, Goldani MZ, et al. Birth mode-dependent association between pre-pregnancy 
maternal weight status and the neonatal intestinal microbiome. Sci Rep. 2016;6(1):1-9.

4. Shabana, Shahid SU, Irfan U. The gut microbiota and its potential role in obesity. Future Microbiol. 2018;13(5):589-603.

5. Su M, Nie Y, Shao R, Duan S, Jiang Y, Wang M, et al. Diversified gut microbiota in newborns of mothers with gestational diabetes mellitus. PLoS One. 2018;13(10):e0205695.

6. Dominguez-Bello MG, De Jesus-Laboy KM, Shen N, Cox LM, Amir A, et al. Partial restoration of the microbiota of cesarean-born infants via vaginal microbial transfer. Nat Med. 2016;22(3):250.

7. Coker MO, Hoen AG, Dade E, Lundgren S, Li Z, Wong AD, et al. Specific class of intrapartum antibiotics relates to maturation of the infant gut microbiota: a prospective cohort study. BJOG-Int J Obstet Gy. 2020;127(2):217-27.

8. Reyman M, van Houten MA, van Baarle D, Bosch AA, Man WH, Chu ML, et al. Impact of delivery mode-associated gut microbiota dynamics on health in the first year of life. Nat Commun. 2019;10(1):1-2.

9. Chu DM, Ma J, Prince AL, Antony KM, Seferovic MD, Aagaard KM. Maturation of the infant microbiome community structure and function across multiple body sites and in relation to mode of delivery. Nat Med. 2017;23(3):314-26.

10. Laursen MF, Bahl MI, Michaelsen KF, Licht TR. First foods and gut microbes. Front Microbiol. 2017;8:356

11. Tanaka M, Nakayama J. Development of the gut microbiota in infancy and its impact on health in later life. Allergol Int. 2017;66(4):515-22.

12. Gérard P. Gut microbiome and obesity. How to prove causality? Ann Am Thorac Soc. 2017;14(Supplement 5):S354-56.

13. Gérard P. Gut microbiota and obesity. Cell Mol Life Sci. 2016;73(1):147-62.

14. Kurilshikov A, Wijmenga C, Fu J, Zhernakova A. Host genetics and gut microbiome: challenges and perspectives. Trends Immunol. 2017;38(9):633-47.

15. Awany D, Allali I, Dalvie S, Hemmings S, Mwaikono KS, Thomford NE, et al. Host and microbiome genome-wide association studies: current state and challenges. Front Genet. 2019;9:637.

16. Calatayud M, Koren O, Collado MC. Maternal Microbiome and Metabolic Health Program Microbiome Development and Health of the Offspring. Trends Endocrin Met. 2019;30:735-44.

17. Wang M, Monaco MH, Donovan SM. Impact of early gut microbiota on immune and metabolic development and function. In Seminars in Fetal and Neonatal Medicine. 2016;21:380-87.

18. Fouhy F, Watkins C, Hill CJ, O'Shea CA, Nagle B, Dempsey EM, et al. Perinatal factors affect the gut microbiota up to four years after birth. Nat Commun. 2019;10(1):1-10

19. Milani C, Duranti S, Bottacini F, Casey E, Turroni F, Mahony J, et al. The first microbial colonizers of the human gut: composition, activities, and health implications of the infant gut microbiota. Microbiol Mol Biol Rev. 2017;81(4):e00036-17.

20. Rinninella E, Raoul P, Cintoni M, Franceschi F, Miggiano GA, Gasbarrini A, et al. What is the healthy gut microbiota composition? A changing ecosystem across age, environment, diet, and diseases. Microorganisms. 2019;7(1):14.

21. Rowland I, Gibson G, Heinken A, Scott K, Swann J, Thiele I, et al. Gut microbiota functions: metabolism of nutrients and other food components. Eur J Nutr. 2018;57(1):1-24.

22. Becattini S, Taur Y, Pamer EG. Antibiotic-induced changes in the intestinal microbiota and disease. Trends Mol Med. 2016;22(6):458-78.
23. Fouhy F, Ross RP, Fitzgerald GF, Stanton C, Cotter PD. Composition of the early intestinal microbiota: knowledge, knowledge gaps and the use of high-throughput sequencing to address these gaps. Gut microbes. 2012;3(3):203-20.

24. Almeida A, Mitchell AL, Boland M, Forster SC, Gloor GB, Tarkowska A, et al. A new genomic blueprint of the human gut microbiota. Nature. 2019;568(7753):499-504.

25. Jovel J, Patterson J, Wang W, Hotte N, O'Keefe S, Mitchel T, et al. Characterization of the gut microbiome using $16 \mathrm{~S}$ or shotgun metagenomics. Front Microbiol. 2016;7:459.

26. Lee PY, Chin SF, Neoh HM, Jamal R. Metaproteomic analysis of human gut microbiota: where are we heading? J Biomed Sci. 2017;24(1):36.

27. Hatch A, Horne J, Toma R, Twibell BL, Somerville KM, Pelle B, et al. A robust metatranscriptomic technology for population-scale studies of diet, gut microbiome, and human health. Int J Genomics. 2019;2019. DOI: https://doi.org/10.1155/2019/1718741

28. Mailhe M, Ricaboni D, Vitton V, Gonzalez JM, Bachar D, Dubourg G, et al. Repertoire of the gut microbiota from stomach to colon using culturomics and next-generation sequencing. BMC Microbiol. 2018;18(1):1-1.

29. Albuquerque D, Stice E, Rodríguez-López R, Manco L, Nóbrega C. Current review of genetics of human obesity: from molecular mechanisms to an evolutionary perspective. Mol Genet Genomics. 2015;290(4):1191-221.

30. Cuevas-Sierra A, Ramos-Lopez O, Riezu-Boj JI, Milagro FI, Martinez JA. Diet, gut microbiota, and obesity: links with host genetics and epigenetics and potential applications. Adv Nutr. 2019;10:S17-S30.

31. Sun L, Ma L, Ma Y, Zhang F, Zhao C, Nie Y. Insights into the role of gut microbiota in obesity: pathogenesis, mechanisms, and therapeutic perspectives. Protein Cell. 2018;9(5):397-403.

32. Ahmad A, Yang W, Chen G, Shafiq M, Javed S, Ali Zaidi SS, Shahid R, Liu C, Bokhari H. Analysis of gut microbiota of obese individuals with type 2 diabetes and healthy individuals. PLoS One. 2019;14(12):e0226372.

33. Ashiq S, Ashiq K, Shabana S, Shahid SU, Qayyum M, Sadia H. Prevalence and role of different risk factors with emphasis on genetics in development of pathophysiology of coronary artery disease (CAD). Pak Heart J. 2020;52(4):279-87.

34. Jin M, Qian Z, Yin J, Xu W, Zhou X. The role of intestinal microbiota in cardiovascular disease. $\mathrm{J}$ Cell Mol Med. 2019;23(4):2343-50.

35. Miele L, Giorgio V, Alberelli MA, De Candia E, Gasbarrini A, Grieco A. Impact of gut microbiota on obesity, diabetes, and cardiovascular disease risk. Curr Cardiol Rep. 2015;17(12):120.

36. Irie J, Itoh H. Dysbiosis in the pathophysiology of coronary artery disease. J Atheroscler Thromb. 2016; 23(8):901-2.

37. Kitai T, Tang WW. Gut microbiota in cardiovascular disease and heart failure. Clin Sci. 2018;132(1): 85-91.

38. Drosos I, Tavridou A, Kolios G. New aspects on the metabolic role of intestinal microbiota in the development of atherosclerosis. Metabolism. 2015;64(4):476-81.

39. Lee P, Yacyshyn BR, Yacyshyn MB. Gut microbiota and obesity: An opportunity to alter obesity through faecal microbiota transplant (FMT). Diabetes Obes Metab. 2019;21(3):479-90.

40. Abenavoli L, Scarpellini E, Colica C, Boccuto L, Salehi B, SharifiRad J, et al. Gut Microbiota and Obesity: A Role for Probiotics. Nutrients. 2019;11(11):2690.

\section{Address for Correspondence:}

Dr. Sana Ashiq, Centre for Applied Molecular Biology (CAMB), University of the Punjab, Lahore-Pakistan Email: sanaashiq72@gmail.com 